www. revistad y o. com

\title{
Las estructuras sociales emergentes: Un complemento de la estructura formal que influye en el rendimiento empresarial en la era COVID-19
}

\author{
Dr. Jorge Armando López-Lemus
}

Recibido: 21 de Noviembre de 2020

Aceptado: 7 de Septiembre de 2021

https://doi.org/10.37610/dyo.v0i75.607

\section{Resumen}

El objetivo principal radica conocer la influencia que ejerce las estructuras sociales emergentes sobre el rendimiento empresarial en las MiPyMES en la era del COVID-19. Se empleó una muestra de 331 participantes pertenecientes estructuras sociales emergentes de las MiPyMES. Los resultados muestran que la estructura social emergente influye positiva y significativamente sobre el rendimiento en la producción y en el rendimiento no financiero. Asimismo, El rendimiento en la producción, influye positiva y significativamente sobre el rendimiento financiero. Finalmente, el rendimiento no financiero influye positiva y significativamente sobre el rendimiento financiero de las MiPyMES en México en la era del COVID-19.

\section{Palabras clave}

Estructura social emergente, Clima Organizacional, Cultura organizacional, Rendimiento Empresarial, COVID-19.

\section{Introducción}

Ante la crisis económica que enfrentan los países derivados por la pandemia del COVID-19, el sector empresarial se ha visto afectado de manera significativa y permanente. Los efectos que ha generado la pandemia han sido de manera inmediata y evidente perjudicado a las organizaciones tras el cierre de las mismas por órdenes gubernamentales (Filimoau, Derqui, \& Matute, 2020; Hall, Scott, \& Gössling, 2020), aún cuando solo haya sido temporal, este tipo de estrategias gubernamentales han puesto en riesgo la economía de las organizaciones al recortar sus ingresos así como la ruptura de las estructuras generadas a través de las cadenas de suministro de los grupos de interés que se han establecido por mucho tiempo (Nicola, et al., 2020)

Ante esta crisis derivada por el COVID-19, las organizaciones de diferentes sectores (Nicola, et al., 2020) han optado en generar diversos cambios estratégicos tanto en su gestión (Brynjolfsson, et al., 2020) como en su estructura (Hao, Xiao, \& Chon, 2020; Kan, Oiwun, \& Chen, 2020) con la finalidad de alcanzar niveles de resiliencia organizacional (Filimoau, Derqui, \& Matute, 2020) que contribuyan en generar su rendimiento empresarial ante la presencia de la pandemia.

\footnotetext{
$凶 \quad$ Dr. Jorge Armando López-Lemus * jorge.lemux@hotmail.com

iD ORCID: 0000-0001-6989-1065

* University of Guanajuato.

Department of Multidisciplinary Studies,

Engineering Division Campus Irapuato-Salamanca

Av. Universidad s/n. Col. Yacatitas,

CP. 38940. Yuriria, Gto. (México)
}

Un estudio realizado por el Foro Económico Mundial señala que, a raíz de la pandemia del COVID-19, las organizaciones han generado estrategias de gestión a distancia e hibridas para mantener su productividad y rendimiento empresarial, sin embargo, para la mayoría de las empresas no están seguros del cómo estas estrategias y cambios organizacionales responderán a largo plazo (Whiting, 2020).

Asimismo, el Kimbrough y Blue (2020) y Whiting (2020) señalan que $78 \%$ de los líderes empresariales consideran que es un problema sumamente importante, debido a que, las estrategias reflejarían un impacto negativo de las nuevas formas de laborar, así como en la productividad y su efecto sobre el rendimiento de las organizaciones. Las razones de este escepticismo radican en los conflictos que se derivan a raíz del estrés que surgen ante nuevas adaptaciones culturales donde la tecnología juega un papel esencial en estos cambios organizacionales, y con ello, afecten el funcionamiento de las empresas (Bonanomi, Hall, Staub-French, Tucker, \& Talamo, 2020; Kimbrough \& Blue, 2020; White, Currie, \& Lockett, 2016)

Ante los nuevos desafíos marcados por el COVID-19, los actores principales de las organizacionales han empleado diferentes métodos de coordinación en paralelo de las estructuras formales como la emergentes para alcanzar los objetivos (Dingel \& Neiman, 2020; Rank, 2008; Stefani \& Torriero, 2013) empresariales. De acuerdo con los estudios realizados por Xiong y King (2018), las dinámicas de las redes informales o emergentes juegan un papel importante para promover el desempeño de las organizaciones por dos motivos, en primer lugar, promueven un trabajo colaborativo mediante un ambiente de confianza y apoyo entre los colaboradores que no estén marcados por la estructura formal, generado un efecto positivo en la cultura y clima 
organizacional. En segundo lugar, promueven el feedback que promueven las estructuras sociales emergentes mediante su liderazgo (White, Currie, \& Lockett, 2016; Xiong \& King, 2018).

El valor y la relevancia del presente estudio se enfoca en la importancia que tienen las estructuras sociales emergentes para el sector empresarial (Efendic \& Ledeneva, 2020) en la época del COVID-19. Es menester que las MiPyMES conozcan, desarrollen y potencialicen nuevas estrategias de gestión internas de la organización, en las que, se alinean actitudes y comportamientos (Terglav, Ruzzier, \& Kaše, 2016) enmarcados por la cultura y el clima organizacional (Efendic \& Ledeneva, 2020; Goebel \& Weißenberger, 2017) así como el liderazgo (Marion, Christiansen, Klar, Schreiber, \& Akif, 2016; Younger \& Fisher, 2020) que ejercen en las estructuras sociales emergentes en las MiPyMES, con la finalidad alcanzar el éxito de la organización en la era COVID-19 (Bauer, Bloch, \& Merkert, 2020) a través de su rendimiento empresarial (Ding \& Rigby, 2011; Goebel \& Weißenberger, 2017) enmarcado por la productividad, rendimiento financiero y no financiero (López-Lemus \& De la Garza, 2019; Noordin R. , Zainuddin, Faud, \& Mail, 2015)

El objetivo principal de la presente investigación radica en conocer y analizar la influencia que ejerce las estructuras sociales emergentes basadas en el liderazgo, clima y cultura organizacional sobre el rendimiento empresarial. En base a ello, se pretende responder a la siguiente pregunta de investigación: ¿la estructura social emergente representa un complemento de la estructura formal que influyen en el rendimiento empresarial en la era COVID-19?

\section{Marco Teórico}

\subsection{Estructura social emergente}

Ante la aparición del COVID-19 a fines del mes de marzo del 2020, se ha especificado a través de la literatura como un parteaguas que inicia una era en la que el COVID-19 juega un papel importante en el desarrollo y funcionamiento de las organizaciones (Sreenivasan \& Suresh, 2021).

Ante la presencia de la pandemia del COVID-19, las organizaciones han tenido en cuenta las bondades que promueven las estructuras que se generan dentro de las empresas. En este sentido, en el interior de las organizaciones se generan dos tipos de estructuras, la formal y la informal (Rank, 2008). Cada una de estas estructuras conforman redes de personas que se desempeñan en línea vertical y horizontal con la finalidad de lograr los objetivos organizacionales.
Figura 1 Estructura organizacional formal. Basado en Dingel y Neiman (2020).

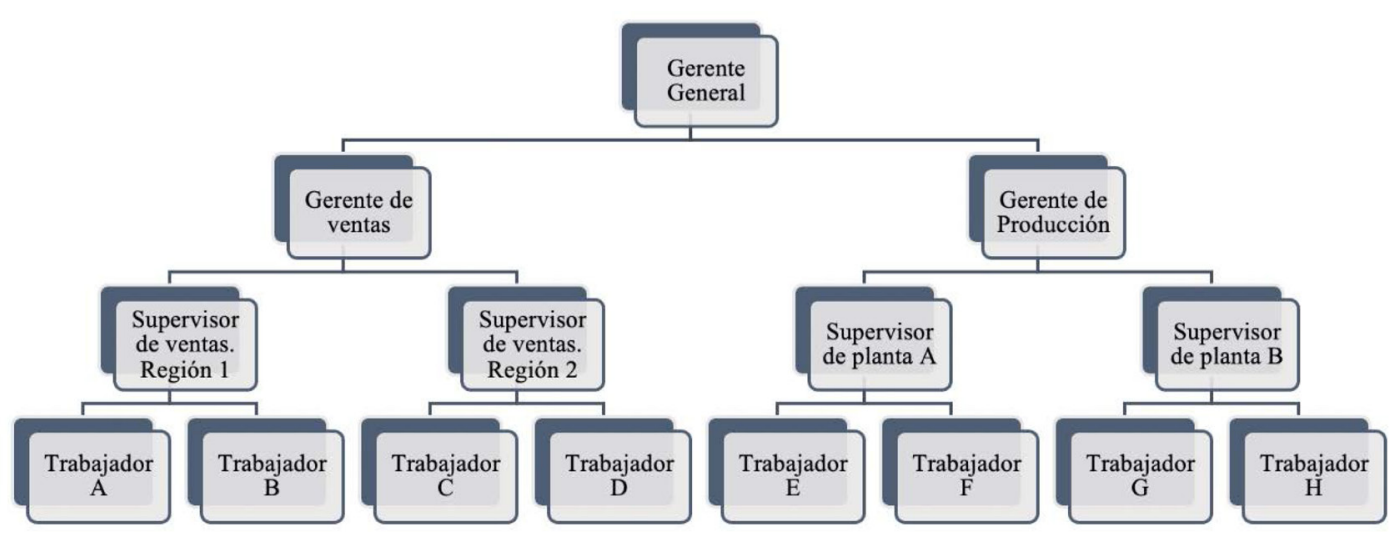


Las estructuras formales son creadas a partir de un organigrama formal (Martínez-León \& MartínezGarcía, 2011) a través de este organigrama se establece la formación y relación de los departamentos generados de manera estratégica (Senge, 1992). Este tipo de estructura especifica la división de trabajo entre los pares que desempeñan el mismo nivel de autoridad y los niveles de gestión (Dipboye, 2018)

La diferenciación horizontal en este tipo de estructuras formales se refiere a la asignación de tareas a los empleados que operan en el mismo nivel jerárquico de la organización, mientras que la diferencia vertical corresponde a la división de la organización en niveles de autoridad (Dipboye, 2018; Sitar \& Škerlavaj, 2018). Estas redes de trabajo son totalmente controladas por la empresa, cuya finalidad radica en gestionar las actividades y procesos para alcanzar los objetivos de la organización. Ver figura 1 .

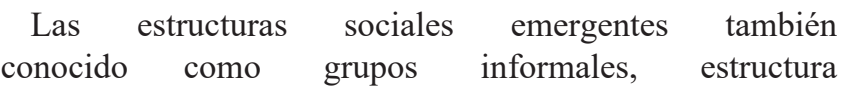

informal o redes informales, es una estructura que surge a partir de la teoría de los roles, y representa un conjunto de personas que se crean a partir de intereses, creencias y actitudes que continuamente se forman a través de las relaciones interpersonales y la conjunción de estos tipos de grupos de diferentes niveles de la organización que conforman una estructura social emergente (Chiavenato, 2004; Dipboye, 2018; Rank, 2008; Sitar \& Škerlavaj, 2018) que surge a partir de la estructura formal (Dipboye, 2018). La cohesión y la eficacia de ambos grupos radica principalmente en la compatibilidad de los miembros (Hussein, 1990; Milgram, 1999; Sallán, 2006).

De acuerdo con Hernández (2003) e Higuita y Leal (2010), la formación de la estructura social emergente en la organización se establece de manera horizontal y vertical, es decir, formación de grupos de personas que pertenecen en una misma área o nivel de jerarquía. Por otro lado, se encuentra la forma vertical, que alude a la formación de grupos informales que son parte de un mismo departamento, pero están ubicados en diferentes niveles jerárquicos de la organización (Dingel \& Neiman, 2020). Ver figura 2.
Figura 2 Estructura organizacional formal. Basado en Dingel y Neiman (2020).

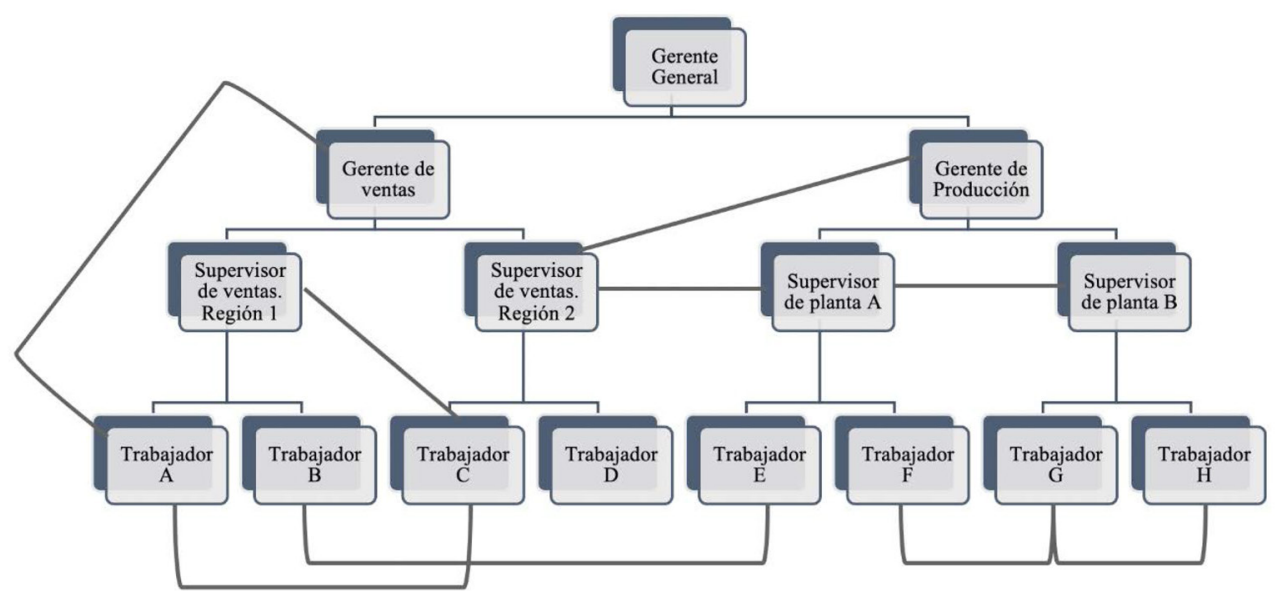


Las estructuras sociales emergentes juegan un papel muy importante dentro de la organización (Chiavenato, 2004) debido a las diversas dinámicas (Viloria, Daza, \& Pérez, 2016) enmarcadas por medio de las creencias y actitudes adaptadas a través de la cultura (Efendic \& Ledeneva, 2020; Goebel \& Weißenberger, 2017; Higuita \& Leal, 2010; Uzkurt, Kumar, Kimzan, \& Eminoğlu, 2013), el ambiente de trabajo promovido por el clima organizacional (Ahmad, Jasimuddin, \& Kee, 2018; Schneider, 2000), así como también, por los comportamientos causados a través de la influencia del líder (Bass \& Avolio, 2004). Es por ello por lo que, estos grupos informales son factores estratégicos en el desempeño de la organización (De Toni \& Nonino, 2010; Värk \& Reino, 2020).

En este sentido, la relevancia de las estructuras sociales emergentes radica a que respondan de manera eficaz y eficiente a través de emplear diferentes métodos de coordinación para alcanzar los objetivos de la organización (Dingel \& Neiman, 2020; Rank, 2008; Stefani \& Torriero, 2013) debido a su fuerte cooperación espontánea y su alta fidelidad al grupo (Higuita \& Leal, 2010). Asimismo, promueven la innovación y aprendizaje en la organización (Bonanomi, Hall, Staub-French, Tucker, \& Talamo, 2020; Värk \& Reino, 2020) en razón a que sus funciones no están delimitadas como los grupos formales (Hartman \& Johson, 1990).

Se debe tener muy en cuenta que las organizaciones son estructuras complejas, y de las cuales el éxito del sector empresarial no solo depende en la capacidad de producir o vender productos o servicios, sino que también, depende de la eficacia del flujo de comunicación y de los vínculos que se generan dentro de las organizaciones (Stefani \& Torriero, 2013). Considerando la relevancia que existe entre los vínculos y las interacciones de las estructuras que se generan dentro de las organizaciones, las actividades y estrategias en la gestión del proceso de trabajo a menudo se generan por medio de las relaciones informales más que la estructura formal (De Toni \& Nonino, 2010; Stefani \& Torriero, 2013; Värk \& Reino, 2020).

En este sentido, los roles de las estructuras sociales emergentes influyen en los procesos de decisión, así como también en el desempeño a nivel individual como en la red de la organización interna (De Toni \& Nonino, 2010) a través del liderazgo, cultura y clima organizacional, factores relevantes en el rendimiento ante la presencia del COVID-19 (Ford \& Marshall, 2020; Newman \& Ford, 2020).

Cultura Organizacional. Considerada como una de las dinámicas de los grupos informales (Chiavenato, 2004) y es definida como el conjunto de valores, creencias y supuestos que comparten las personas que laboran dentro de una organización (Cameron \& Quinn, 1999). La cultura organizacional es uno de los factores que proporciona la información necesaria para el funcionamiento de la organización, así como también los comportamientos de los grupos formales e informales (Uzkurt, Kumar, Kimzan, \& Eminoğlu, 2013).

Clima Organizacional. Es considerada como una principales de las dinámicas de las estructuras sociales emergentes (Chiavenato, 2004) definida como la percepción de los empleados hacia las políticas, prácticas y procedimientos que ocurren un entorno laboral en el que se presentan y se desarrollan las actitudes y comportamientos individuales y grupales en las organizaciones (Ahmad, Jasimuddin, \& Kee, 2018; Schneider, 2000) generando así, un ambiente laboral que puede ser en beneficio o en perjuicio de la organización.

Liderazgo. Es definida como el proceso en el que una persona influye en otras a través de su comportamiento, creencia y valores con la finalidad de lograr objetivos personales o de la propia organización y que, a su vez, potencialice el crecimiento y adaptación dinámica, así como la permanencia de los involucrados, en este sentido, del grupo de seguidores pertenecientes a grupos formales o informales (Bass \& Avolio, 2004; López-Lemus, 2016). Este factor resulta ser una de las dinámicas más importantes en las estructuras sociales emergentes (Chiavenato, 2004) debido a la influencia que ejercen en las estructuras organizacionales tanto formal como informal.

Ante la aparición de la pandemia del COVID-19, tanto directivos, gerentes como empleados se han visto en la necesidad colaborar de forma remota (Ford \& Marshall, 2020), es decir, han empleado la tecnología para realizar sus funciones a través de la gestión de equipos virtuales (Newman \& Ford, 2020) generando una nueva cultura y clima organizacional que respalde su fuerza laboral tanto virtual, presencial o mixta y con ello, reactivar la productividad de la organización ante escenarios emergentes provocados por la pandemia del COVID-19. Considerando que el efecto de la pandemia es un fenómeno que permanecerá durante un tiempo considerable, es necesario que los líderes de las organizaciones mejoren el rendimiento y el compromiso de los equipos de trabajo a través de establecer y mantener una cultura y clima de confianza a través de las herramientas de comunicación formal e informal para liderar a los miembros del equipo, y con ello, generar estrategias para lograr la rentabilidad y sustentabilidad de la organización a través del rendimiento empresarial en la era del COVID-19 (Ford \& Marshall, 2020; Hoch \& Dulebohn, 2017).

En base a lo anterior, se busca ayudar a los miembros de la organización a potencializar sus fortalezas y capacidades para lograr objetivos personales o colectivos a través de las estructuras sociales emergentes o informales. Es por ello por lo que, se considera al liderazgo como uno de los principales factores que fomenta una cultura en la que se promuevan ideas, participación entre las personas que conforman el grupo de trabajo, así como un clima favorable en el desarrollo de sus funciones colaborativas dentro de la organización. 
En este sentido, las dinámicas de las estructuras sociales emergentes basadas en la cultura y clima organizacional así como el liderazgo, generan un grado de cohesión sumamente importante debido a que logran influir a través en el equipo de trabajo (Higuita \& Leal, 2010; Hussein, 1990; Viloria, Daza, \& Pérez, 2016) y con ello, potencializar la productividad, aumentar el rendimiento empresarial (Noordin R. , Zainuddin, Faud, Mail, \& Kaziemah, 2015) convirtiéndose en una ventaja competitiva empresarial (Peng, 2012; VargasHernández, Guerra, Bojórquez, \& Bojórquez, 2014) en la que es empleada para enfrentar los desafíos enmarcados por la pandemia del COVID-19 con la finalidad de reactivar la economía de la organización ante escenarios emergentes.

\subsection{Rendimiento Empresarial}

Ante la crisis económica que enfrenta el sector empresarial derivado de la pandemia del COVID-19, el rendimiento de las organizaciones ha sido afectada de manera significativa y parmente. Para reducir este problema organizacional, el sector empresarial público y privado, han desarrollado diversas estrategias enfocadas en las dinámicas de las estructuras sociales emergentes para evitar a toda costa ser perjudicadas en su rendimiento productivo, financiero y no financiero (Ahmad, Jasimuddin, \& Kee, 2018; Noordin R. , Zainuddin, Faud, Mail, \& Kaziemah, 2015) ante decisiones gubernamentales establecidas para evitar la propagación del virus del COVID-19 en entornos organizacionales (Filimoau, Derqui, \& Matute, 2020; Hall, Scott, \& Gössling, 2020).

En investigaciones recientes se ha demostrado que existe una relación significativa entre las dinámicas de las estructuras sociales emergentes y el desempeño de las organizaciones. Sin embargo, la mayoría de los estudios realizados en la literatura solo adopta una dimisión para construir las estructuras sociales emergentes, así como un factor unidimensional en el rendimiento organizacional (Ding \& Rigby, 2011), lo que limita la posibilidad de diferenciar la influencia de los diferentes rendimientos que cuentan las organizaciones (Ahmad, Jasimuddin, \& Kee, 2018; Noordin R., Zainuddin, Faud, Mail, \& Kaziemah, 2015).

Por otro lado, las organizaciones pueden diferir entre sí por su estructura, tamaño, ingresos, negocios, etc., (Akhtaruzzaman, Boubaker, \& Sensoy, 2020; Ding \& Rigby, 2011), la presente investigación refleja algunas diferencias significativas con las que se han realizado en la literatura, debido a que, se estudia la relación de las estructuras sociales emergentes de las organizaciones partir del liderazgo, clima y cultura organizacinal (Ford \& Marshall, 2020; Hoch \& Dulebohn, 2017) en relación con el rendimiento empresarial enfocado en el rendimiento en la producción, financiero y no financiero (Ahmad, Jasimuddin, \& Kee, 2018; Noordin R. , Zainuddin, Faud, Mail, \& Kaziemah, 2015) de las MiPyMES en la era del COVID-19.
De acuerdo con Newman y Ford (2020) los líderes de las estructuras sociales emergentes, así como los directivos y gerentes empresariales logran tener rendimiento en las organizaciones a medida que ponen en énfasis adicional establecer objetivos, así como habilidades de gestión del desempeño basadas en el liderazgo (Osland, et al., 2020), cultura y clima organizacional (Efendic \& Ledeneva, 2020; Goebel \& Weißenberger, 2017). Davila (2000) considera que, uno de los principales objetivos de las organizaciones del sector empresarial está enfocada en potencializar su rendimiento por medio de los resultados obtenidos a través de la empresa (Schendel \& Hoper, 1979).

Asimismo, Noordin, Zainuddin, Faud, y Mail, (2015) señalan que el rendimiento empresarial está enfocado a través del grado en que la empresa alcanza el éxito por medio del rendimiento en la producción, financiero y no financiero (Akhtaruzzaman, Boubaker, \& Sensoy, 2020; Ding \& Rigby, 2011). De acuerdo con Ahmad y Kee (2018), Akhtaruzzaman, et al., (2020), Hoque y James, (2000), Noordin, Zainuddin, Faud, y Mail, (2015) y Schabek (2020) estos tres factores del rendimiento empresarial son definidos de la siguiente manera:

El rendimiento en la producción. se refiere a la tendencia que las organizaciones implementan todas aquellas prácticas, herramientas de gestión dentro de su función operativa (González-Benito, 2016). En este sentido, se hace énfasis al desempeño de las empresas en relación con los competidores principales en términos de participación de mercado, volumen de ventas generadas, así como la productividad de la empresa (Ahmad K. \& Kee, 2018; Noordin, Zainuddin, Faud, \& Mail, 2015).

El rendimiento financiero. es un indicador de rentabilidad, que es empleado para medir la efectividad del rendimiento de las empresas, con la finalidad de controlar los costos y gastos y de esta manera convertir las ventas en utilidades (Ortiz, 2004). En este sentido se hace énfasis a los indicadores financieros como el grado de utilidad generada por medio del retorno de inversión (ROI), así como también, el grado de rentabilidad generada sobre los recursos propios de la empresa (ROE) (Akhtaruzzaman, Boubaker, \& Sensoy, 2020; Hoque \& James, 2000; Noordin , Zainuddin, Faud, \& Mail, 2015; Schabek, 2020).

El rendimiento no financiero. es aquella que se genera a través de la superioridad que ha alcanzado una empresa sobre sus competidores en el mercado que participa enfocado en calidad, ventaja competitiva de costos, volumen de ventas, entrega a tiempo, satisfacción del cliente, así como la cuota del mercado (Akhtaruzzaman, Boubaker, \& Sensoy, 2020; Hoque \& James, 2000; Noordin R. , Zainuddin, Faud, Mail, \& Kaziemah, 2015) 
Estas dimensiones del rendimiento de la empresa permitirá a los líderes y empresarios de las MiPyMES tomar decisiones que contribuirán en analizar y generar estrategias para alcanzar los objetivos organizacionales considerando a las estructuras sociales emergentes como uno de los factores clave para lograr el rendimiento de las empresas así como alcanzar el éxito a través de la mejora continua en la medición del rendimiento de las MiPyMES (Deming, 1986; Ding \& Rigby, 2011; Flynn, Schroeder, \& Sakakibara, 1994) en la era del COVID-19.
A través variables estudiadas en la presente investigación, se establecieron las siguientes hipótesis, H1: las estructuras sociales emergentes influyen positivamente sobre el rendimiento en la producción, H2: las estructuras sociales emergentes influyen positivamente sobre el rendimiento no financiero, H3: el rendimiento en la producción influye positiva y significativamente sobre el rendimiento financiero. H4: el rendimiento no financiero influye positiva y significativamente sobre el rendimiento financiero. Ver figura 3.
Figura 3 Modelo SEM hipotético puesto en cuestión. Diseño propio. Nota: En la figura 1 se muestran las hipótesis enumeradas conforme a su orden, mismas que, se establecen de la presente investigación

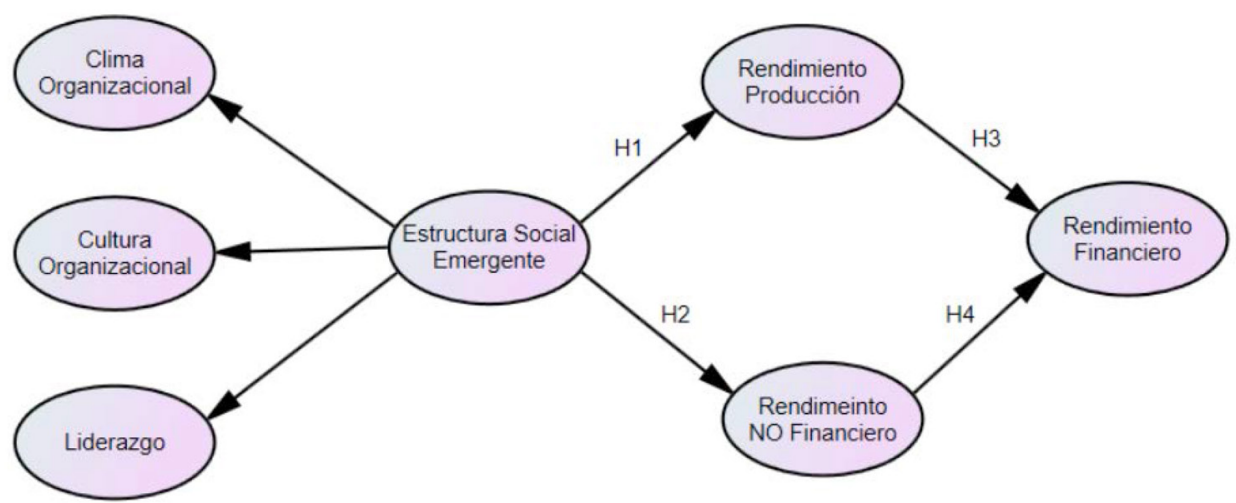

\section{Metodología}

La metodología empleada en la investigación fue cuantitativa ya que se utilizaron instrumentos que permitieron medir cada una de las variables seleccionadas para este estudio, Del mismo modo, fue de tipo explicativa en razón que se pretende dar una visión general y aproximada del comportamiento de las variables, en este sentido, las estructuras sociales emergentes basadas en el liderazgo, clima y cultura organizacional y el rendimiento en la producción, financiero y no financiero de las MiPyMES del estado de Guanajuato. México. Asimismo, fue observacional ya que se pretendió describir el fenómeno sin ninguna manipulación o intervención en las variables que determinaron el proceso de la presente investigación. En cuanto al tipo metodológico, fue transversal ya que se aplicaron instrumentos cuantitativos en un solo momento a los participantes del estudio con la finalidad de recolectar la información de las variables para su análisis y medición.

\subsection{Muestra}

El tipo de muestreo aplicado en la investigación fue no probabilístico intencional debido a que se pretendió obtener el mayor número de participantes en el estudio. En cuanto a los criterios de inclusión, fueron empleados que conformaban una estructura social emergente o grupo informal y que contara con una antigüedad de al menos un año laborando en la empresa. Para el levantamiento de la información, se diseñó una a través de una plataforma alojado a un servidor de internet en el que los sujetos del estudio ingresaron para contestar los instrumentos para el análisis cuantitativa de la presente investigación.

De esta manera, se logró obtener una muestra de 331 participantes. De los cuales, el 60.1\% $(n=199)$ fueron mujeres mientras que el 39.9\% ( $n=132)$ fueron hombres. En cuanto a la edad de los participantes, el 20.2\% $(n=67)$ cuenta con una edad menor a los 20 años, el $26.6 \%(n=88)$ tiene una edad de entre 21 a 30 años, el 27.2\% ( $n=90)$ cuenta con una edad de entre 31 a 40 años, el $23.9 \%(n=79)$ tiene una edad de entre 41 a 50 años, el $2.1(\mathrm{n}=7)$ cuenta con una edad superior a los 50 años. En cuanto a la escolaridad de los participantes, el $25.4 \%(n=84)$ cuenta con un nivel de escolaridad hasta bachillerato, el $69.8 \% \quad(n=231)$ cuenta con estudios de licenciatura, el $4.8 \%(\mathrm{n}=16)$ cuenta con un posgrado. En cuanto al sector de las MiPyMES, el 30.2\% $(n=100)$ son de comercio, el $21.1 \%(n=70)$ con de servicios, mientras que el $48.6 \%(n=161)$ corresponden al sector industrial.

Para identificar el tamaño de las empresas, se consideró la estratificación del tamaño de empresas en México a través del número de empleados de acuerdo con el Diario Oficial de la Federación (DOF, 2009). En este sentido, el 20.5\% ( $n=68)$ fueron micro, el $25.1 \%(n=83)$ fueron pequeñas, el $20.2 \%$ $(n=67)$ fueron medianas mientras que el $34.1 \% \quad(n=113)$ fueron grandes empresas. 
Para el análisis de los datos obtenidos, se empleó el software estadístico SPSS v.21 y para la comprobación de las hipótesis establecidas, se desarrolló un modelo de ecuación estructural (SEM), a través del software estadístico Amos v.21.

Asimismo, se procedió a realizar una correlación entre las variables latentes estudiadas. De esta manera, se demuestra que existe una relación positiva y significativa (Bonett \& Wright, 2000; Pearson, 1929; Pearson, 1931) entre las variables latentes: estructuras sociales emergentes (clima y cultura organizacional, así como también el liderazgo) y el rendimiento en la producción, financiero y no financiero, medida a través del coeficiente de correlación de Pearson como se muestra en la tabla 1 .
Tabla 1 Estadística descriptiva: Promedios, desviación estándar, varianza, y correlación de las variables.

\begin{tabular}{|c|c|c|c|c|c|c|c|c|c|}
\hline VARIABLES & Media & D.S. & Varianza & 1 & 2 & 3 & 4 & 5 & 6 \\
\hline Clima Organizacional & 3.6 & 0.61 & 0.38 & 1 & & & & & \\
\hline Cultura Organizacional & 4.2 & 0.75 & 0.56 & $0.46^{* *}$ & 1 & & & & \\
\hline Liderazgo & 3.9 & 0.64 & 0.42 & $0.47^{* *}$ & $0.43^{* *}$ & 1 & & & \\
\hline Rendimiento Producción & 5.2 & 1.23 & 0.42 & $0.43^{* *}$ & $0.50^{* *}$ & $0.40^{* *}$ & 1 & & \\
\hline Rendimiento No Financiero & 5.2 & 1.22 & 1.53 & $0.42^{* *}$ & $0.54^{* *}$ & $0.40^{* *}$ & $0.70^{* *}$ & 1 & \\
\hline Rendimiento Financiero & 5.0 & 1.32 & 1.75 & $0.41^{* *}$ & $0.42^{* *}$ & $0.35^{* *}$ & $0.75^{* *}$ & $0.64^{* *}$ & 1 \\
\hline
\end{tabular}
${ }^{* *} p<0.001$.

Nota: En la tabla 1 se muestran los estadísticos descriptivos, así como la correlación entre las variables liderazgo, clima y cultura organizacional, como de las estructuras sociales emergentes, así como el rendimiento empresarial basado en los factores del rendimiento en la producción, no financiero y financiero. En este sentido, se puede apreciar que la correlación entre las variables oscila de entre 0.40 a 0.75 positiva y significativa (Bonett \& Wright, 2000; Pearson, 1929; Pearson, 1931).

\subsection{Confiabilidad y Validez de Instrumentos}

De acuerdo con los análisis de las variables manifiestas de las variables latentes, no siguieron una distribución normal. Sin embargo, de acuerdo con Bollen y Stine (1992) y Hair et al. (2017) las técnicas de bootstrapping representan un método para llevar a cabo la corrección de normalidad, por un lado, problemas originados por el tamaño de muestra y por otro, que los datos no sigan una distribución normal. Asimismo, las técnicas de bootstrapping proporciona un valor de significancia (p) sin asumir la normalidad de la muestra y corrige el valor probabilístico proporcionado por el método de máxima verosimilitud para contrastar el ajuste global (Davison \& Hinkley, 1997; Efron \& Tibshirani, 1993; Hair, Hult, Ringle, \& Sarstedt, 2017)

Para la validez de los instrumentos utilizados, se consideró la validez de constructo debido a que es el principal de los diferentes tipos de validez. En este sentido, "la validez de constructo es el concepto unificador que integra las consideraciones de validez de contenido y de criterio en un marco común para probar hipótesis acerca de relaciones teóricamente relevantes" (Messick, 1980; p.1015). Asimismo, Cronbach (1984) considera que "la meta final de la validación es la explicación y comprensión y, por tanto, esto nos lleva a considerar que toda validación es validación de constructo" (p.126). 


\subsubsection{Estructura Social Emergente}

Para medir este constructo, se emplearon tres instrumentos correspondientes al Clima Organizacional (Schneider \& Bartlett, 1970), Cultura Organizacional (Yiing \& Ahmad, 2006) y Liderazgo (Castro, Lupano, Benatuil, \& Nader, 2007) mismas que representan las principales dinámicas de las estructuras sociales emergentes (Viloria, Daza, \& Pérez, 2016).

3.2.1.1 Clima Organizacional. Para medir esta variable se empleó la dimensión de conflictos inter-grupos de la escala de clima organizacional de Schneider y Bartlett (1970). La dimensión está conformada por 5 ítems y emplean una escala tipo Likert de 5 puntos done 1 representa "Nunca" y 5 "Siempre". Para evaluar la confiabilidad del instrumento se empleó el Alfa de Cronbach a través $(\propto=0.701)$, misma que resultó satisfactorio (Cronbach, 1951; Hair, Hult, Ringle, \& Sarstedt, 2014)

En cuanto a la validez del instrumento de innovación, se desarrolló un CFA basado en Schneider y Bartlett (1970) a través de un modelo de ecuación estructural. Para la validación del SEM, se consideró la prueba de Ji-cuadrado $\left(x^{2}=10.675\right.$ / $\mathrm{gl}=4)$, el índice comparativo de ajuste (CFI=0.981), el índice de Tucker-Lewis (TLI=0.953) y el Índice de la Aproximación de la raíz cuadrada del error cuadrático medio (RMSEA=0.07), por lo que resultaron ser satisfactorios (Bollen, 1989; Jöreskog \& Sörbom, 1981; López-Lemus \& Zavala, 2019; Muthén, 2002; Rigdon, 1996). Asimismo, se comprobó la validez convergente a través de las cargas factoriales estandarizadas $(\lambda)$ de las variables observables mismas que fueron superiores a .40 (Hair, Hult, Ringle, \& Sarstedt, 2017; López-Lemus \& Zavala, 2019; Jöreskog \& Sörbom, 1981) con un alto nivel de significancia. Ver tabla 2.
Tabla 2 Cargas factoriales estandarizadas y Alfa de Cronbach del Clima Organizacional. Fuente: Modelo basado en Schneidery Bartlett (1970).

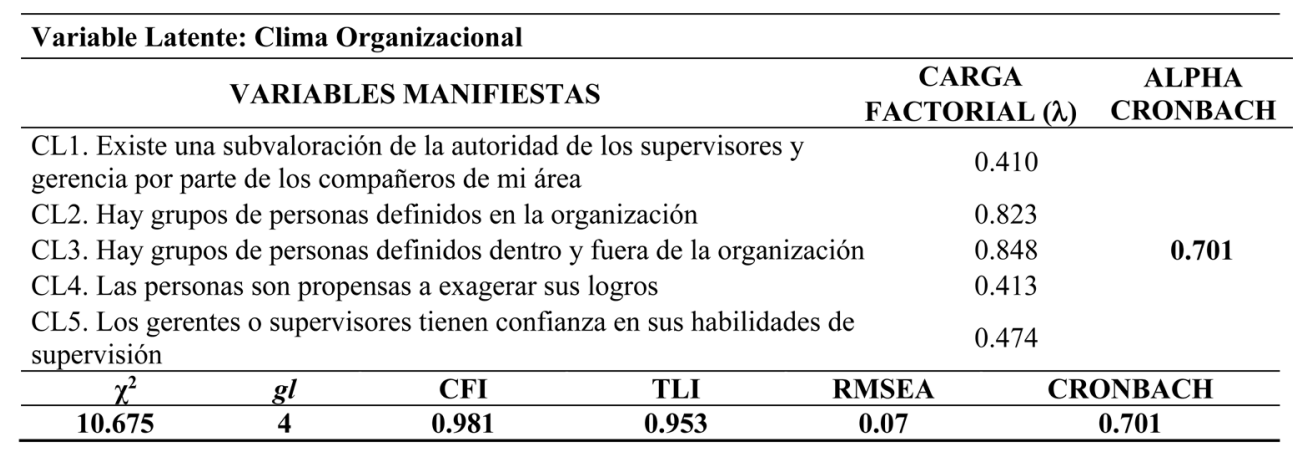

Nota: En la tabla 2 se muestran las cargas factoriales en $1^{\circ}$ orden del constructo clima organizacional. En este sentido las cargas factoriales de $1^{\circ}$ orden la variable de la latente oscila de entre 0.410 a 0.848. Cargas que resultaron ser positivas y significativas. Del mismo modo, la consistencia interna del instrumento medida a través del Alfa de Cronbach resultó ser satisfactoria ( $a=0.701)$. En cuanto a los índices de bondad de ajuste ( $X 2=10.675 \mathrm{gl}=4 ; \mathrm{CFI}=0.981$; $T L I=0.953 ;$ RMSEA=0.07) del modelo resultaron ser aceptables (Bollen, 1989; Hair, Hult, Ringle, \& Sarstedt, 2017; Jöreskog \& Sörbom, 1981; López-Lemus \& Zavala, 2019; Muthén \& Muthén, 1998-2007; Muthén, 2002; Rigdon, 1996).

3.2.1.2 Cultura Organizacional. Para medir este constructo se empleó la escala de cultura organizacional desarrollado por Yiing y Ahmad (2006). Este instrumento está conformado a través de 6 ítems y emplea una escala de Likert de 7 puntos, donde 1 representa "Nunca" y 5 "Siempre". Para evaluar la confiabilidad de este instrumento se empleó el Alfa de Cronbach para el instrumento $(\alpha=0.888)$, mismo que resultó satisfactorio (Cronbach, 1951; Hair, Hult, Ringle, \& Sarstedt, 2014).

En cuanto a la validez del instrumento, se desarrolló un CFA basado en Yiing y Ahmad (2006), a través de un modelo de ecuación estructural. Para la validación del SEM, se consideraron los índices de bondad y ajuste del modelo $\left(x^{2}=26.843 / \mathrm{gl}=9 ; \mathrm{CFI}=0.983 ; \mathrm{TLI}=0.972 ; \mathrm{RMSEA}=0.07\right)$ por lo que resultó ser satisfactorio (Bollen, 1989; Jöreskog \& Sörbom, 1981). Asimismo, se comprobó la validez convergente a través de las cargas factoriales estandarizadas $(\lambda)$ de las variables observables mismas que fueron superiores a .40 (Hair, Hult, Ringle, \& Sarstedt, 2017; López-Lemus \& Zavala, 2019; Jöreskog \& Sörbom, 1981) con un alto nivel de significancia. Ver tabla 3. 
Tabla 3 Cargas factoriales estandarizadas y Alfa de Cronbach de la Cultura Organizacional Fuente: Modelo basado en Yiing y Ahmad (2006).

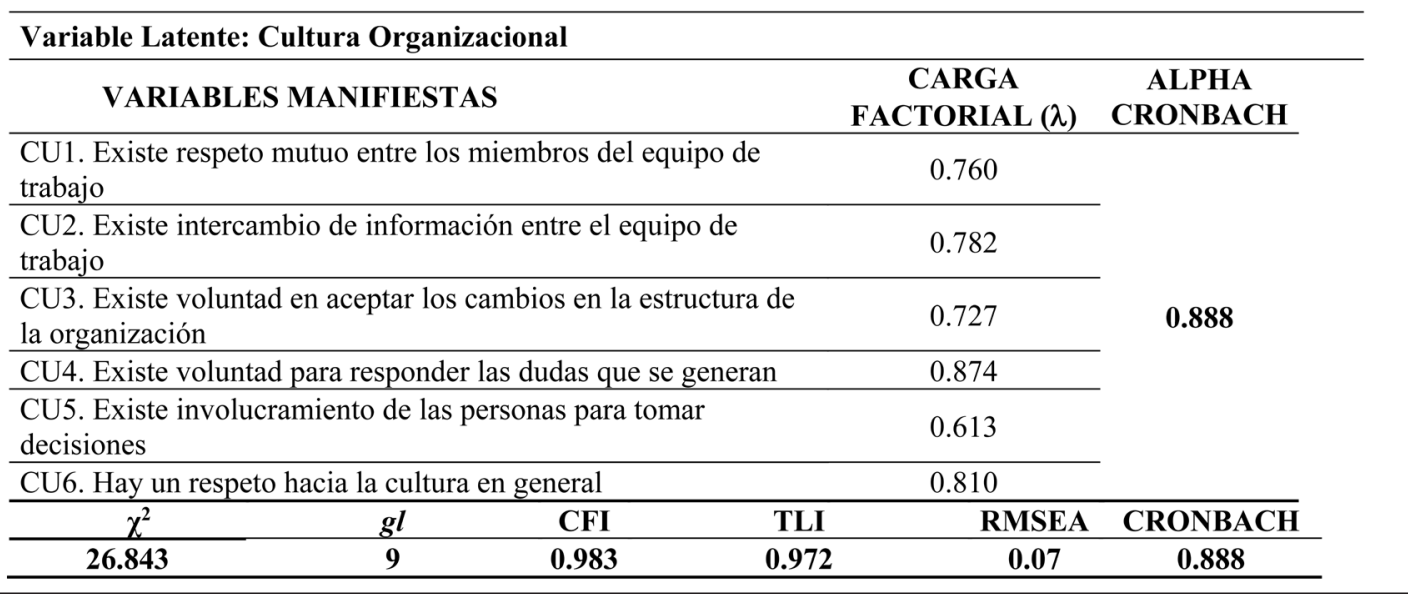

Nota: En la tabla 3 se muestran las cargas factoriales en $1^{\circ}$ orden del constructo cultura organizacional. En este sentido las cargas factoriales de $1^{\circ}$ orden la variable de la latente oscila de entre 0.613 a 0.874. Cargas que resultaron ser positivas y significativas. Del mismo modo, la consistencia interna del instrumento medida a través del Alfa de Cronbach resultó ser satisfactoria ( $\alpha=0.888)$. En cuanto a los índices de bondad de ajuste ( $\chi 2=26.843 \mathrm{gl}=9 ; \mathrm{CFI}=0.983$; $T L I=0.972 ;$ RMSEA=0.07) del modelo resultaron ser aceptables (Bollen, 1989; Hair, Hult, Ringle, \& Sarstedt, 2017; Jöreskog \& Sörbom, 1981; López-Lemus \& Zavala, 2019; Muthén \& Muthén, 1998-2007; Muthén, 2002; Rigdon, 1996).

3.2.1.3 Liderazgo. Para medir este constructo, se emplearon dos dimensiones correspondientes al liderazgo (1) transformacional y (2) transaccional del instrumento CELID-A de Castro, Lupano, Benatuil y Nader (2007), basado en el MQL (Multifactor Leadership Questionnaire) de Bass y Avolio (2004). Este instrumento considera por 28 ítems entre ambos contractos. Los reactivos emplean un formato tipo Likert con 5 puntos de respuesta, en donde 1 representa "Total desacuerdo con la afirmación" y 5 "Total acuerdo con la afirmación". Para evaluar la confiabilidad de este instrumento se empleó el Alfa de Cronbach (a) para las dimensiones de liderazgo transformacional $(\alpha=0.901)$ y transaccional ( $a=0.794)$, así como para ambos constructos que representan el liderazgo $(a=0.893)$, mismas que resultaron satisfactorias (Cronbach, 1951; Hair, Hult, Ringle, \& Sarstedt, 2014).

En cuanto a la validez del instrumento del liderazgo basadas en dos constructos (Transformacional y Transaccional) del CELID-A, se desarrolló un análisis factorial confirmatorio (CFA) de segundo orden basado en Castro, Lupano, Benatuil y Nader (2007) a través de un modelo de ecuación estructural. Para la validación del SEM, se consideraron los índices de bondad y ajuste del modelo $\left(x^{2}=236.389 / \mathrm{gl}=85 ; \mathrm{CFI}=0.937\right.$; $\mathrm{TLI}=0.922$; RMSEA $=0.07$ ) por lo que los índices de bondad y ajuste del modelo resultaron ser satisfactorios (Bollen, 1989; Jöreskog \& Sörbom, 1981; López-Lemus \& Zavala, 2019; Muthén, 2002; Rigdon, 1996). Asimismo, se comprobó la validez convergente a través de las cargas factoriales estandarizadas $(\lambda)$ de las variables observables mismas que fueron superiores a .40 (Hair, Hult, Ringle, \& Sarstedt, 2017; López-Lemus \& Zavala, 2019; Jöreskog \& Sörbom, 1981) con un alto nivel de significancia. De acuerdo con lo señalado por los autores, se eliminaron 13 ítems debido a que no tuvieron una carga factorial satisfactoria (Muthén, 2002) por lo que el instrumento se conformó por 15 ítems. ver tabla 4. 
Tabla 4 Cargas factoriales estandarizadas y Alfa de Cronbach del Liderazgo Fuente: Modelo basado en Castro, Lupano, Benatuily Nader (2007).

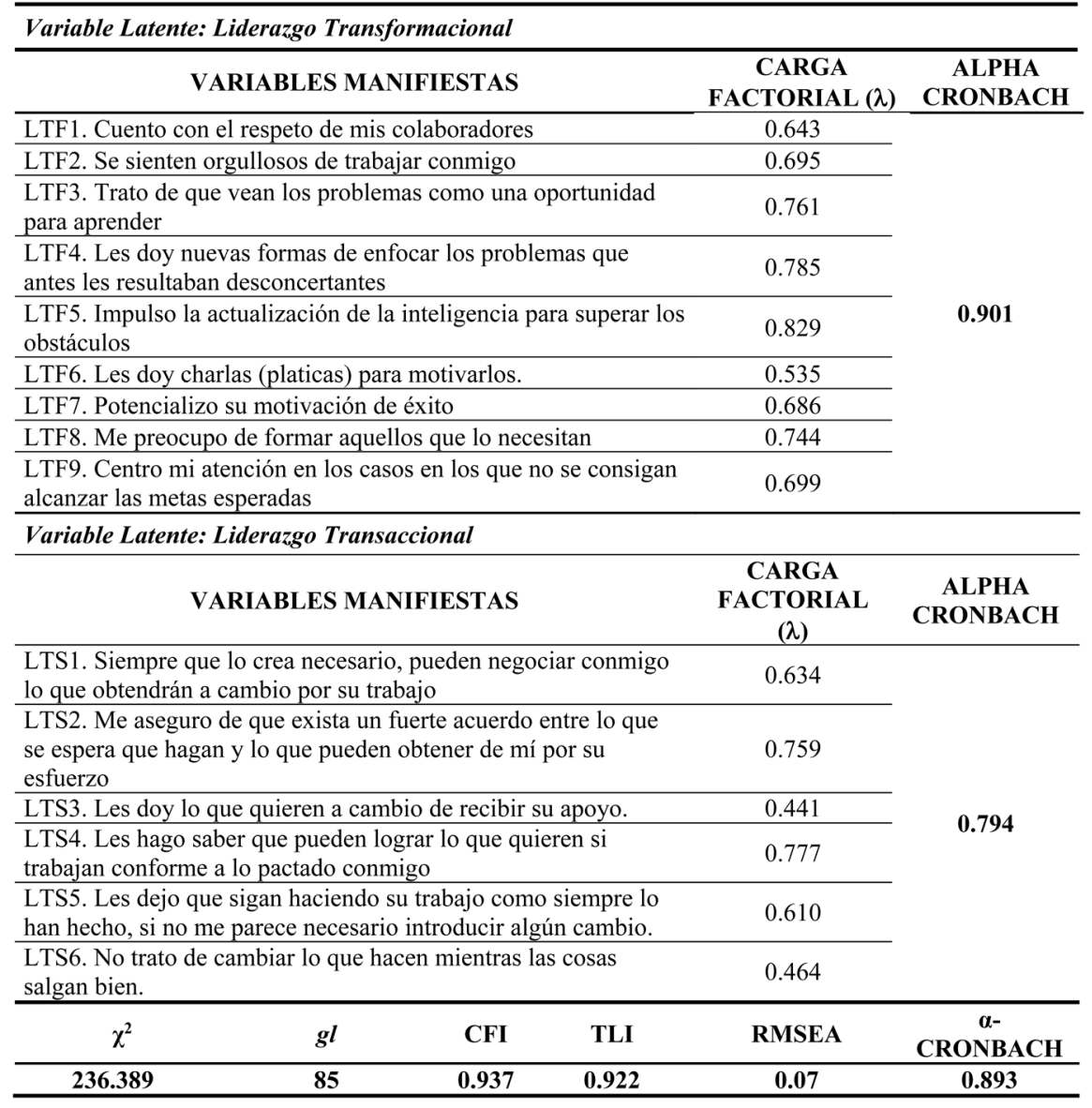

Nota: En la tabla 4 se muestran las cargas factoriales en $1^{\circ}$ orden del constructo del liderazgo. En este sentido las cargas factoriales de $1^{\circ}$ orden la variable del latente liderazgo transformacional oscila de entre 0.535 a 0.829, para el liderazgo transaccional cuenta con cargas factoriales de 0.441 a 0.759 . Así mismo, todas las cargas factoriales de las dos dimensiones del constructo liderazgo resultaron ser positivas y significativas. Del mismo modo, la consistencia interna del instrumento medida a través del Alfa de Cronbach resultó ser satisfactoria para el liderazgo transformacional ( $a=0.901)$ y transaccional ( $a=0.794)$ como también para todo el instrumento ( $\alpha=0.893$ ). En cuanto a los índices de bondad de ajuste $(X 2=236.389 \mathrm{gl}=85 ; C F I=0.937 ; T L I=0.922 ; R M S E A=0.07)$ del modelo resultaron ser aceptables (Bollen, 1989; Hair, Hult, Ringle, \& Sarstedt, 2017; Jöreskog \& Sörbom, 1981; López-Lemus \& Zavala, 2019; Muthén \& Muthén, 19982007; Muthén, 2002; Rigdon, 1996)

\subsubsection{Rendimiento Empresarial}

Se empleó la validación del instrumento de rendimiento empresarial de Noordin, Zainuddin, Faud, y Mail (2015). Este instrumento considera tres dimensiones: (1) rendimiento en la producción, (2) financiero y (3) no financiero. El instrumento se conforma por 12 ítems y emplea una escala de Likert de 7 puntos, donde 1 representa "pobre" y 5 "excelente". Para evaluar la confiabilidad de este instrumento se empleó el Alfa de Cronbach para el rendimiento en (1) la producción $(\alpha=0.952)$, financiero $(\alpha=0.913)$ y no financiero $(\alpha=0.873)$, así mismo se calculó para el instrumento en general $(a=0.949)$, mismo que resultó satisfactorio (Cronbach, 1951; Hair, Hult, Ringle, \& Sarstedt, 2014).
En cuanto a la validez del instrumento del rendimiento empresarial, se desarrolló un CFA en $2^{\circ}$ orden basado en Noordin, Zainuddin, Faud, y Mail (2015), a través de un modelo de ecuación estructural. Para la validación del SEM, se consideraron los índices de bondad y ajuste del modelo $\left(x^{2}=163.830 / \mathrm{gl}=62 ; \mathrm{CFI}=0.973 ; \mathrm{TLI}=0.966\right.$; RMSEA=0.07) por lo que resultó ser muy satisfactorio (Bollen, 1989; Jöreskog \& Sörbom, 1981; López-Lemus \& Zavala, 2019; Muthén, 2002; Rigdon, 1996). Asimismo, se comprobó la validez convergente a través de las cargas factoriales estandarizadas $(\lambda)$ de las variables observables mismas que fueron superiores a .40 (Hair, Hult, Ringle, \& Sarstedt, 2017; López-Lemus \& Zavala, 2019; Jöreskog \& Sörbom, 1981) con un alto nivel de significancia. Ver tabla 5. 
Tabla 5 Cargas factoriales estandarizadas y Alfa de Cronbach del Rendimiento Empresarial

Fuente: Basado en Noordin Zainuddin, Faud y Mail (2015).

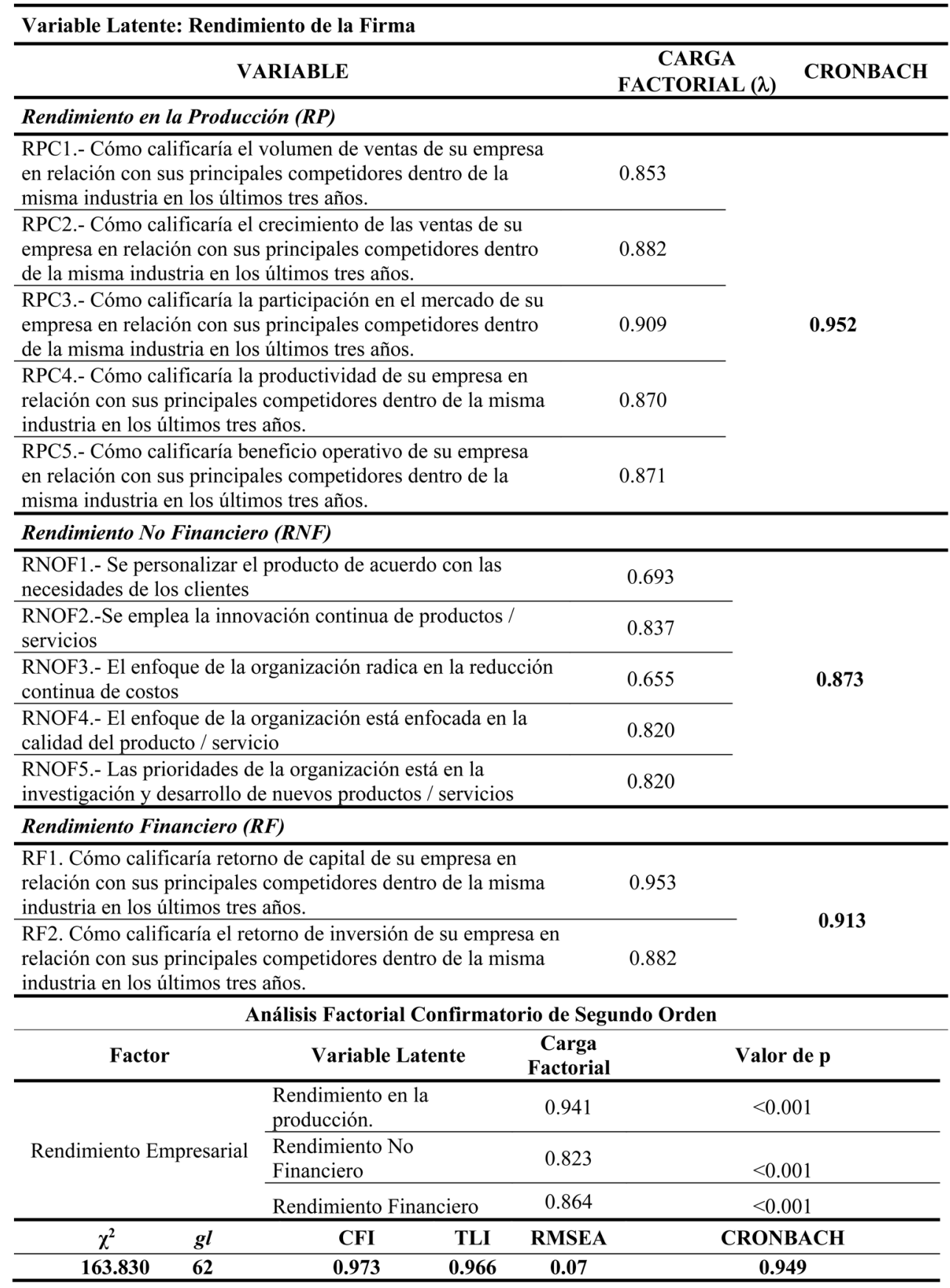

Nota: En la tabla 5 se muestran las cargas factoriales en $1^{\circ}$ y $2^{\circ}$ orden del constructo del rendimiento empresarial. En este sentido las cargas factoriales de $1^{\circ}$ orden la variable del latente rendimiento en la producción y comercialización oscilan de entre 0.853 a 0.909 , para el rendimiento no financiero cuenta con cargas factoriales de 0.655 a 0.837 mientras que las cargas del rendimiento financiero resultaron ser de 0.882 y 0.953. Así mismo, todas las cargas factoriales de las tres dimensiones del constructo rendimiento de la firma resultaron ser positivas y significativas. En cuanto a las cargas factoriales de $2^{\circ}$ orden, tanto el rendimiento en la producción $(0.941 ; p<0.01)$, no financiero $(0.823 ; p<0.01)$ y el financiero $(0.864 ; p<0.01)$ son positivas y significativas. De la misma forma, la consistencia interna del instrumento medida a través del Alfa de Cronbach resultó ser satisfactoria para las dimensiones rendimiento en la producción ( $a=0.952)$, financiero ( $a=0.873$ ) y no financiero ( $a=0.913)$ como también para todo el instrumento ( $a=0.949)$. En cuanto a los índices de bondad de ajuste (X2=163.830 gl=62; CFI=0.973; TLI=0.966; RMSEA=0.07) del modelo resultaron ser aceptables (Bollen, 1989; Hair, Hult, Ringle, \& Sarstedt, 2017; Jöreskog \& Sörbom, 1981; López-Lemus \& Zavala, 2019; Muthén \& Muthén, 1998-2007; Muthén, 2002; Rigdon, 1996). 


\section{Resultados y Conclusiones}

Para evaluar el modelo de ecuación estructural (SEM) hipotético bajo la técnica de Bootstrapping y el método de robustez máxima verosimilitud (ML) (Satorra \& Bentler, 1994) considerando un remuestreo de 1,000 Bootstraps. Para la validación del modelo, se consideró la prueba de Ji-Cuadrado $\left(x^{2}=569.11 / \mathrm{gl}=267 ; x^{2} / \mathrm{gl}=2.13 ; \mathrm{p}<0.001\right)$, el Índice de Ajuste Comparativo (CFI=0.94), Índice de Ajuste Normalizado (NFI=0.91), el Índice de Tucker-Lewis $(\mathrm{TLI}=0.93)$, Índice de Bondad de Ajuste $(\mathrm{GFI}=0.96)$, Índice de Bondad de Ajuste Ajustado (AGFI=0.91), índice de la aproximación de la Raíz Cuadrada del Error Cuadrático Medio (RMSEA=0.05) y el Residuo Cuadrático Medio Estandarizado $(\mathrm{SRMR}=0.06)$ por lo que los índices de bondad y ajuste del modelo SEM resultaron ser satisfactorios (Bollen, 1989; Hair, Hult, Ringle, \& Sarstedt, 2017; Jöreskog \& Sörbom, 1981; López-Lemus \& Zavala, 2019; Muthén \& Muthén, 1998-2007; Rigdon, 1996; Tucker \& Lewis, 1973) ver figura 4.
Figura 4 Cargas

estructurales estandarizadas

del modelo SEM. Diseño

propio

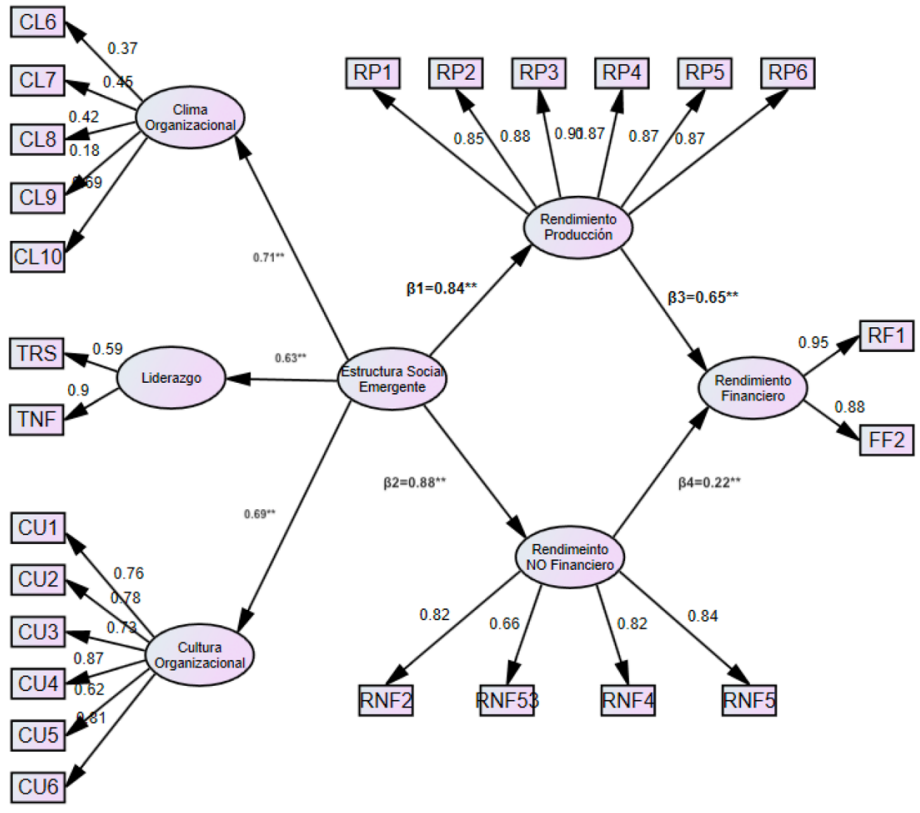

Nota: En la figura 3 se muestran las cargas estructurales estandarizadas de las variables exógenas sobre las endógenas y en base a las cargas ( $\beta$ ) se analizan cada una de ellas para evaluar las hipótesis establecidas en la investigación. En este sentido, el análisis SEM muestra cargas de entre 0.220 ( $\beta_{4}$ ) y $0.88\left(\beta_{2}\right)$, mismas que son positivas y significativas ( $\left.p<0.001\right)$. Asimismo, los índices de bondad de ajuste del modelo SEM (X2=589.11 gl=267; $C F I=0.94 ;$ $N F I=0.91 ; T L I=0.93 ; G F I=0.96 ; A G F I=0.91 ; R M S E A=0.05 ;$ SRMR=0.06) resultaron ser satisfactorios (Bollen, 1989; Hair, Hult, Ringle, \& Sarstedt, 2017; Jöreskog \& Sörbom, 1981; López-Lemus \& Zavala, 2019; Muthén \& Muthén, 1998-2007; Rigdon, 1996; Tucker \& Lewis, 1973).

Para evaluar las hipótesis establecidas en la presente investigación, se recurrió a los resultados obtenidos a través de las cargas estructurales del modelo SEM (ver figura 3). En este sentido, para evaluar la $\mathrm{H}_{1}$ : las estructuras sociales emergentes influyen positiva y significativamente sobre el rendimiento en la producción, se evaluó la carga estructural especificada a través del sendero de $\beta_{1}$. En base al análisis y evaluación de esta, se puede afirmar que, la estructura social emergente influye $\left(\beta_{1}=0.84 ; \mathrm{p}<0.001\right)$ de manera positiva y significativa sobre el rendimiento en la producción. Por lo tanto, la hipótesis $\mathrm{H}_{1}$ es aceptada.

En este sentido, la estructura social emergente se genera a través de la interacción laboral y los intereses, creencias y actitudes mediante las relaciones interpersonales, así como la conjunción de los tipos de grupos de diferentes niveles de la organización (Dipboye, 2018; Rank, 2008; Sitar \& Škerlavaj, 2018) influye positivamente sobre las prácticas operativas y prácticas así como en las herramientas de gestión para su función, generando relaciones interpersonales en las que desarrollan lazos de cooperación y sinergia para promover el desempeño y productividad tanto en lo individual como en lo colectivo para potencializar la productividad de la empresa (Ahmad, Jasimuddin, \& Kee, 2018; Filimoau, Derqui, \& Matute, 2020; Ford \& Marshall, 2020; González-Benito, 2016; Hall, Scott, \& Gössling, 2020; Hoch \& Dulebohn, 2017; Noordin R. , Zainuddin, Faud, \& Mail, 2015) como respuesta a las estrategias que establecen las organizaciones para enfrentar la pandemia del COVID-19

Para evaluar la $\mathrm{H}_{2}$ : las estructuras sociales emergentes influyen positivamente sobre el rendimiento no financiero, se analizó la carga estructural $\beta_{2}$ del modelo SEM hipotético. En base a la carga estructural, se comprueba estadísticamente que la estructura social emergente influye $\left(\beta_{2}=0.88 ; \mathrm{p}<0.001\right)$ positiva y significativamente sobre el rendimiento no financiero. De esta forma, la hipótesis $\mathrm{H}_{2}$ establecida es aceptada. 
Por lo tanto, las estructuras sociales juegan un factor sumamente importante en las organizaciones (Brynjolfsson, et al., 2020), debido a que, las dinámicas enmarcadas por el liderazgo, la cultura y clima organizacional favorece en la cooperación proactiva entre los miembros del equipo de trabajo (Ahmad, Jasimuddin, \& Kee, 2018) en tiempos del COVID-19, por lo que, éstas dinámicas de las estructuras sociales emergentes potencializan el servicio y calidad de los productos o servicios, promoviendo una sinergia entre las estructuras formales e informales (Efendic \& Ledeneva, 2020; Goebel \& Weißenberger, 2017; Hao, Xiao, \& Chon, 2020; Kan, Oiwun, \& Chen, 2020).

Esta estrategia representa una ventaja competitiva debido a que asume diversas acciones colaborativas que promueven la baja de costos operacionales, y con ello, el aumento de las ventas y la entrega a tiempo, así mismo, se incrementa la cuota de mercado (Akhtaruzzaman, Boubaker, \& Sensoy, 2020; Hoque \& James, 2000; Noordin R. , Zainuddin, Faud, Mail, \& Kaziemah, 2015) en las organizaciones en escenarios turbulentos promovidos por le COVID-19. En este sentido, se convierte en un área de oportunidad para emplear estas estructuras sociales emergentes (Hao, Xiao, \& Chon, 2020; Kan, Oiwun, \& Chen, 2020) como una estrategia que beneficiará a la resiliencia empresarial (Filimoau, Derqui, \& Matute, 2020) promoviendo su reactivación económica mediante el rendimiento de la organización (Värk \& Reino, 2020)

De la misma forma, para evaluar la hipótesis $\mathrm{H}_{3}$ : el rendimiento en la producción influye positiva $\mathrm{y}$ significativamente sobre el rendimiento financiero, se analizó la carga estructural $\beta_{3}$. De acuerdo con la evaluación de esta carga, se cuenta con suficiente evidencia estadística para afirmar que el rendimiento de la producción influye $\left(\beta_{3}=0.65 ; \mathrm{p}<0.001\right)$ positiva y significativamente en el rendimiento financiero de las empresas. Por lo que, la hipótesis $\mathrm{H}_{3}$, es aceptada.

Este hallazgo es relevante debido a que, el rendimiento de la producción que promueven las estructuras sociales emergentes a través de las prácticas y gestión operativa en la productividad individual y colectiva de la organización (Ahmad, Jasimuddin, \& Kee, 2018; Filimoau, Derqui, \& Matute, 2020; Ford \& Marshall, 2020; González-Benito, 2016; Hall, Scott, \& Gössling, 2020; Hoch \& Dulebohn, 2017; Noordin R. , Zainuddin, Faud, \& Mail, 2015) potencializará la rentabilidad empresarial, la utilidad generada a través del retorno de inversión y el grado de rentabilidad que promueven los recursos propios de la empresa (Akhtaruzzaman, et al., 2020; Hoque \& James, 2000; Noordin, et al., 2015; Schabek, 2020). Por lo que, las estructuras sociales emergentes son factores clave para promover un rendimiento financiero del sector empresarial ante situaciones adversas del mercado provocadas por la pandemia del COVID-19.
Finalmente, para evaluar la $\mathrm{H}_{4}$ : el rendimiento no financiero influye positiva y significativamente sobre el rendimiento financiero. se evaluó el resultado de la carga estructura $\beta_{4}$. De acuerdo con el resultado de la carga estructural a través del modelo SEM, se comprueba que el rendimiento no financiero influye $\left(\beta_{4}=0.22 ; \mathrm{p}<0.001\right)$ positiva y significativamente sobre el rendimiento financiero. En este sentido, se procede aceptar la hipótesis $\mathrm{H}_{4}$.

La relevancia del hallazgo es sumamente importante debido a que, una de las principales dinámicas que establecen las estructuras sociales emergentes radica en la sinergia que promueven los miembros de la estructura (Viloria, Daza, \& Pérez, 2016) pertenecientes a diferentes niveles de la organización (Bonanomi, Hall, Staub-French, Tucker, \& Talamo, 2020; Dingel \& Neiman, 2020; Dipboye, 2018; Efendic \& Ledeneva, 2020; Sitar \& Škerlavaj, 2018) en generar una ventaja competitiva basada en bajos costos, entrega a tiempo y satisfacción del cliente (Ahmad, Jasimuddin, \& Kee, 2018), con la finalidad de alcanzar los objetivos empresariales (Ford \& Marshall, 2020; Newman $\&$ Ford, 2020), de manera que, el rendimiento no financiero (Noordin R. , Zainuddin, Faud, \& Mail, 2015; Schabek, 2020) promovido por las estructuras sociales emergentes potencializan las utilidades generadas por medio del retorno de inversión así como la rentabilidad y rendimiento de las empresas (Akhtaruzzaman, Boubaker, \& Sensoy, 2020; Goebel \& Weißenberger, 2017; Soto, 2020) como una de las principales estrategias que contribuye a la sostenibilidad de la empresa. En este sentido, las estructuras sociales emergentes representan un complemento de la estructura formal (Milgram, 1999; Stefani \& Torriero, 2013) que influyen en el rendimiento empresarial en la era COVID-19 (Bauer, Bloch, \& Merkert, 2020; Filimoau, Derqui, \& Matute, 2020; Hall, Scott, \& Gössling, 2020; Hao, Xiao, \& Chon, 2020; Nicola, et al., 2020).

La contribución de este estudio es pertinente y de gran valor debido a que evalúa el efecto de las estructuras sociales emergentes que influyen en el rendimiento en la producción, financiero y no financiero del sector empresarial en la era del COVID-19. Algunas investigaciones han demostrado la importancia de todas estas variables para la gestión en el sector empresarial de manera separada, pero nunca juntas bajo un contexto de un evento disruptivo global como lo es el COVID-19. Se argumenta que este estudio puede, por lo tanto, ser utilizado como un proxy para comprender mejor el efecto acumulativo de algunos de los principales determinantes del rendimiento empresarial efectivo y el empleo en las MiPyMES, lo cual es para modelar el futuro de las organizaciones y con ello, lograr la resiliencia ante desastres y crisis económicos. 
Asimismo, es menester que los líderes empresariales tengan presente que tanto estructuras sociales formales como emergentes sufrirán diversos cambios enfocados al liderazgo, clima y cultura de la organización debido a que, diversos estudios han señalado que la era posterior al COVID-19 serán bajo modelos de trabajo híbridos (PatakiBittó \& Kapusy, 2021) y en base a esta nueva modalidad, el comportamiento de las estructuras tanto formales como informales o emergentes generarán nuevas estrategias de liderar y con ello, nuevas formas de establecer un clima y una cultura organizacional que pueda contribuir al rendimiento de las organizaciones.

Ante este fenómeno es importante señalar que, ante las nuevas tendencias laborales del pre-COVID-19, los grupos estructurales estarán enfocados a ámbitos laborales presenciales y en línea (on-line) lo que se conoce como híbrido. Esta estrategia que actualmente emplean las organizaciones contrae consigo una serie de brechas de investigación debido a que estas nuevas tendencias organizacionales contrae nuevas formas y estructuras que se van generando a medida que se pretenden lograr los resultados de las empresas.

Ante las nuevas modalidades que el sector empresarial se encuentra adoptando bajo un enfoque hibrido para hacer frente a la pandemia del COVID-19 es menester poner atención a las nuevas tendencias de organización laboral en las que juegan las estructuras organizacionales tanto formales como emergentes para identificar los beneficios que atraen estas estructuras con la finalidad de generar estrategias para poder subsistir en un mercado altamente competitivo. En este sentido, el modelo hipotético propuesto aconseja que las organizaciones deben poner atención en el clima y cultura organizacional, así como el liderazgo de las estructuras sociales emergentes con la finalidad de garantizar el rendimiento empresarial, y con ello, mantener el bienestar de los empleados, así como la rentabilidad y sostenibilidad organizacional (Haque, 2021).

Es de suma importancia señalar que, los impactos en la salud en los eventos de una pandemia no se aíslan en un solo país, sino que representan en la inserción de procesos mucho más amplios de urbanización, globalización, cambio ambiental, y capitalismo contemporáneo (Allen, et al., 2017; Hall, Scott, \& Gössling, 2020). En este sentido, el sector empresarial debe tener claro que las estructuras organizacionales juegan un papel importante en su rendimiento y posicionamiento en un mercado vulnerable a una pandemia como lo es el COVID-19.

Cabe señalar que, existen cuatro nuevos coronavirus (HCoV-229E, HCoV-OC43, HCoV-NL63, HCoV-HKU1) endémicos en las poblaciones humanas y hay otros tres coronavirus identificados como SARS-CoV, MERS-CoV y COVID-19 (Berry, Gamieldien, \& Fielding, 2015; Hall, Scott, \& Gössling, 2020; Rothan \& Byrareddy, 2020; Wu, Nethery, Sabath, Braun, \& Dominici, 2020), siendo este ultimo el que ha marcado cambios y afectaciones sustanciales los seres humanos y por consecuencia en las organizaciones a nivel mundial. Asimismo, es pertinente considerar que, muy a pesar de que varios países, entre ellos México, han sumado esfuerzos para su reactivación económica a través de la vacunación masiva de sus ciudadanos contra el COVID-19, se han presentado variaciones del COVID-19 identificados como Delta y Delta plus, estas variantes son consideradas como nuevas amenazas para lograr la reactivación económica del sector empresarial. Por tanto, es necesario que las organizaciones generen estrategias a través de las estructuras sociales emergentes como las formales que le permita a la organización ser resiliente en un mercado emergente y amenazado por la pandemia del COVID-19.

Es necesario puntualizar que, aparte del COVID-19 y sus variantes Delta y Delta Plus, aún no se han registrado brotes de otros tipos de virus o variaciones, sin embargo, es muy probable que se estarán presentando en alguna población a corto o mediano plazo por las variaciones que ha tenido el COVID-19, y con ello, las afectaciones a las organizaciones (Soto, 2020) y mercados nacionales e internacionales. Ante esta preocupación, es necesario generar estrategias de gestión que contribuyan a generar resiliencia en el sector empresarial encontrando nuevas áreas de oportunidades a través de las estructuras sociales emergentes que puedan dar respuesta ante la presencia de estas pandemias que, muy seguramente, surgirán en nuestra población en poco tiempo.

Las limitaciones del presente estudio radican en que es un estudio de corte transversal en los que se emplearon las estructuras sociales emergentes como objeto de estudio durante la pandemia del COVID-19, se sugiere que se realicen investigaciones referentes a las variables analizadas tanto de las estructuras formales e informales mediante métodos de corte longitudinal en los que se puedan identificar las diferencias del comportamiento de las variables antes, durante y después de la pandemia del COVID-19 así como la forma en el que va cambiando con respecto al nivel que pertenecen los grupos de las estructuras formales y no complementada con las estructuras sociales emergentes, con la finalidad de generar estrategias que potencialicen a las organizaciones para alcanzar la sustentabilidad y rendimiento de las empresas y con ello, generar nuevas líneas de investigación que contribuyen a la generación de conocimiento diferentes áreas.

\section{Referencias}

Ahmad K., J. S., \& Kee, W. (2018). Organizational climate and job satisfaction: do employees' personalities matter? Management Decision, 56 (2). pp. pp.421-440. Doi: 10.1108/MD-10-2016-0713. 
Akhtaruzzaman, M., Boubaker, S., \& Sensoy, A. (2020). Financial contagion during COVID-19 crisis. Finance Research Letters, pp. 1-20. Doi: 10.1016/j. frl.2020.101604.

Allen, T., Murray, K., Zambrana-Torrelio, C., Morse, S., Rondinini, C., Di Marco, M., . . . Daszak, P. (2017). Global hotspots and correlates of emerging zoonotic diseases. Nature Communications, 8(1), pp. 1-10. Doi: 10.1038/s41467-017-00923-8.

Baker, M. (2020). Who cannot work from home? Characterizing occupations facing increased risk during the COVID-19 pandemic using 2018 BLS data. The preprint Server for Healt Science, pp. 1-18. Doi: 10.1101/2020.03.21.20031336.

Bass, B., \& Avolio, B. (2004). Multifactor Leadership Questionnaire. Third Edition Manual and Sampler Set. MindGarden, Inc. .

Bauer, L., Bloch, D., \& Merkert, R. (2020). Ultra Long-Haul: An emerging business model accelerated by COVID-19. Journal of Air Transport Management , 89. pp. 1-8. Doi: 10.1016/j.jairtraman.2020.101901.

Berry, M., Gamieldien, J., \& Fielding, B. (2015). Identification of new respiratory viruses in the new millennium. . Viruses, 7(3), pp. 996-1019. Doi: 10.3390/ v7030996.

Bollen, K. (1989). Structural equations with latent variables. New York, NYS : John Wiley \& Sons.

Bonanomi, M., Hall, D., Staub-French, S., Tucker, A., \& Talamo, C. (2020). The impact of digital transformation on formal and informal organizational structures of large architecture Engineering. Construction and Architectural Management , 27(4), pp. 872-892. Doi: 10.1108/ECAM-03-2019-0119.

Bonett, D., \& Wright, T. (2000). Simple size requirements for estimating Pearson, Kendall and Sperman correlations. . Psichometrika, 65(1). pp. 23-28. Doi: 10.1007/ BF02294183.

Brynjolfsson, E., Horton, J., Ozimek, A., Rock, D., Sharma, G., \& Ye, H. (2020, 11 4). COVID-19 and Remote Work: An Early Look at US Data. Retrieved from https://www.voced.edu.au/handle/ngv:86288

Cameron, K., \& Quinn, R. (1999). Diagnosing and Changing Organizational Culture. Based on the Competing Values Framework. Reading, MA.: Addison-Wesley,
Castro, A., Lupano, M., Benatuil, D., \& Nader, M. (2007). Teoría y evaluación del liderazgo. Buenos Aires, Argentina: Paidós.

Chiavenato, I. (2004). Comportamiento Organizacional: la dinámica del éxito en las organizaciones. México: Thomson.

Cronbach, L. (1951). Coefficient alpha and internal structure of test. Psycho-metrica, 16. pp. 297 - 335. Doi: 10.1007/ BF02310555.

Davison, A., \& Hinkley, D. (1997). Bootstrap Methods and Their Application. Cambridge University Press: Cambridge.

De Toni, A., \& Nonino, F. (2010). The key roles in the informal organization: a net- work perspective analysis. The Learning Organization , 17(1), pp. 86-103. Doi: 10.1108/09696471011008260.

Deming, W. (1986). Out of the Crisis. Cambridge, MA.: MIT Press: .

Ding, H., \& Rigby, J. (2011). An Investigation on the Relationship between Informal Networks and Organizational Performance. En I. R. (eds), Internationalization, Design and Global Development. IDGD 2011 (págs. pp 490-493. Doi: 10.1007/978-3642-21660-2_55). Springer, Berlin, Heidelberg.

Dingel, J., \& Neiman, B. (2020). How Many Jobs Can be Done at Home? NBER: National Breau of Economic Research, pp. 1-18. Doi: 10.3386/w26948.

Dipboye, R. (2018). Social Structures in Organizations . The Emerald Review of Industrial and Organizational Psychology, pp. 315-367. Doi: 10.1108/978-1-78743785-220181010 .

DOF. (30 de 06 de 2009). Diario Oficial de la Federación. Obtenido de Acuerdo por el cual se establece la estratificación de la micro, pequeñas y medianas empresas.:

http://dof.gob.mx/nota_detalle_popup. php?codigo=5096849

Efendic, A., \& Ledeneva, A. (2020). The importance of being networked: The costs of informal networking in the Western Balkans region. Economic Systems, pp. 1-44. Doi: 10.1016/j.ecosys.2020.100784.

Efron, B., \& Tibshirani, R. (1993). An Introduction to the Bootstrap. . New York.: Chapman Hall. 
Filimoau, V., Derqui, B., \& Matute, J. (2020). The COVID-19 pandemic and organisational commitment of senior hotel managers. International Journal of Hospitality Management, 91, pp. 1-13. Doi: 10.1016/j. ijhm.2020.102659.

Flynn, B., Schroeder, R., \& Sakakibara, S. (1994). A Framework for Quality Management Research and an Associated Measurement Instrument. Journal of Operations Management, , 11(4), pp. 339-366. Doi:10.1016/S0272-6963(97)90004-8.

Ford, R., \& Marshall, G. (2020). Virtual team leader communication: employee perception and organizational reality. International Journal of Business Communication, 57(4), pp. 452-473. Doi: 10.1177/2329488419829895.

Goebel, S., \& Weißenberger, B. (2017). The Relationship Between Informal Controls, Ethical Work Climates, and Organizational Performance. J Bus Ethics, 141. pp. 505-528. Doi: 10.1007/s10551-015-2700-7.

González-Benito, J. (2016). A study of the effect of manufacturing proactivity on business performance. International Journal of Operations \& Production Management, 25(3), pp. 222 - 241. Doi: $10.1108 / 01443570510581844$.

Hair, j., Hult, G., Ringle, C., \& Sarstedt, M. (2014). A primer on partial least squares structural equation modeling (PLS SEM). USA: SAGE Piblications. USA.

Hair, J., Hult, G., Ringle, C., \& Sarstedt, M. (2017). A Primer on Partial Least Squares Structural Equation Modeling (PLS-SEM). 2nd Ed. Sage: Thousand Oaks.

Hall, C., Scott, D., \& Gössling, S. (2020). Pandemics, transformations and tourism: Be careful what you wish for. Tourism Geographies, pp. 577-598. Doi: 10.1080/14616688.2020.1759131.

Hao, F., Xiao, Q., \& Chon, K. (2020). COVID-19 and China's Hotel Industry: Impacts, a Disaster Management Framework, and Post-Pandemic Agenda. International Journal of Hospitality Management, 90. pp. 1-11. Doi: 10.1016/j.ijhm.2020.102636.

Haque, A. (2021). The COVID-19 pandemic and the role of responsible leadership in health care: thinking beyond employee well-being and organisational sustainability. Leadership in Health Services, 34(1). pp. 52-68. Doi:10.1108/LHS-09-2020-0071.
Hartman, R., \& Johson, J. (1990). Formal and informal group comunication structure: An examination of their relationship to role ambiguity. Social Networks, 12 (2). pp. 127-151. Doi:10.1016/0378-8733(90)90002-Q.

Higuita, D., \& Leal, J. (2010). Microculturas y cultura organziacional: Construccion dialéctica en la organización. Revista Facultad de Ciencias Económicas: Investigación y Reflexión, 18 (1). pp. 151-167. .

Hoch, J., \& Dulebohn, J. (2017). Team personality composition, emergent leadership and shared leadership in virtual teams: A theoretical framework. Human Resource Management Review, 27(4), pp. 678-693. Doi: 10.1016/j.hrmr.2016.12.012.

Hoque, Z., \& James, W. (2000). Linking balanced score card measures to size market factors: Impact on organizational performance. Journal of Management Research, 12. pp. 1-17. Doi: 10.2308/jmar.2000.12.1.1.

Hussein, R. (1990). Understanding and Managing Informal Groups. Management Decision, 28 (8). pp. 36-41. Doi: 10.1108/00251749010000038.

Jöreskog, K., \& Sörbom, D. (1981). LISREL: Analysis of linear structural relationships by maximum likelihood and least squares methods. Chicago, IL:: National Educational Resources.

Kan, A., Oiwun, J., \& Chen, Z. (2020). Promoting effectiveness of "working from home": findings from Hong Kong working population under COVID-19. Asian Education and Development Studies, pp.1-19. Doi: 10.1108/AEDS-06-2020-0139.

Kimbrough, K., \& Blue, A. (21 de 10 de 2020). Tu próximo cambio de trabajo podría ser más fácil de lo que piensas. Recuperado el 5 de 11 de 2020, de Tu próximo cambio de trabajo podría ser más fácil de lo que piensas

López-Lemus, J. (2016). Recursos personales del líder y su influencia con el estilo de liderazgo. . México.: Unpublished $\mathrm{PhD}$. Thesis. Universidad de la Salle, Bajío. .

López-Lemus, J., \& De la Garza, M. (2019). The practices of business management, innovation and entrepreneurship: influencing factors in the performance of entrepreneur firms. NovaScientia, 22(11(1)), pp.: 357-383. Doi: 10.21640/ns.v11i22.1795. 
López-Lemus, J., \& Zavala, M. (2019). Validación del inventario de habilidaddes políticas de Ferris mediante análisis factorial de segundo orden. En D. Vargas, Aspectos metodológicos para la investigación social: Modelos de ecuaciones estructurales (págs. pp. 153175). Ciudad de México: UNAM.

Marion, R., Christiansen, J., Klar, H., Schreiber, C., \& Akif, M. (2016). Informal leadership, interaction, cliques and productive capacity in organizations: A collectivist analysis. The Leadership Quarterly, 27(2), pp. 242-260. Doi: 10.1016/j.leaqua.2016.01.003.

Martínez-León, I., \& Martínez-García, J. (2011). "The influence of organizational structure on organizational learning. International Journal of Manpower, 32 (5/6), pp.537-566. Doi: 10.1108/01437721111158198.

McEvily, B., Soda, G., \& Tortoriello, M. (2014). More formally: rediscovering the missing link between formal organization and informal social structure. The Academy of Management Annals, 8(1), pp. 299-345.

Milgram, L. (1999). Differentiating Formal and Informal Groups. Managing teams, pp. 151.

Muthén, B. (2002). Beyond SEM: General latent variable modeling. Behaviometrika, 29(1), 81-117. Doi: 10.2333/ bhmk.29.81.

Muthén, B., \& Muthén, L. (1998-2007). Mplus Version 5.0 statistical analysis with latent variables: User's Guide. Los Angeles, CA. USA: Fourth ed. Muthen \& Muthen.

Newman, S., \& Ford, R. (2020). Five Steps to Leading Your Team in the Virtual COVID-19 Workplace. Organizational Dynamics, pp. 1-11. Doi:10.1016/j. orgdyn.2020.100802.

Nicola, M., Alsafi, Z., Sohrabi, C., Kerwan, A., Al-Jabir, A., Losifidis, C., . . . 2020. (2020). The socio-economic implications of the coronavirus pandemic (COVID-19): A review. International Journal of Surgery, 78, pp. 185193. Doi: 10.1016/j.ijsu.2020.04.018.

Noordin, R., Zainuddin, Y., Faud, \& Mail, R. (2015). Performance outcomes of strategic management accounting information usage in Malasia: Insights from electrical and electronics companies. Procedia Economics and Finance, 31. pp. 13-25. Doi: 10.1016/ S2212-5671(15)01127-2.

Ortiz, A. (2004). Análisis Financiero Aplicado y principios de administración Financiera. (12a Edición. ed.). Bogotá. Colombia: Departamento de Publicaciones. Universidad Externado de Colombia.
Osland, M., Mendenhall, B., Reiche, B., Szkudlarek, R., Bolden, P., Courtice, V., . . Shi, A. (2020). Perspectives on Global Leadership and the COVID-19 Crisis. In J. Osland, B. Szkudlarek, M. Mendenhall, \& B. (. Reiche (Eds.), Advances in Global Leadership (Advances in Global Leadership (pp. pp. 3-56. Doi: 10.1108/S1535120320200000013001). Emerald Publishing Limited.

Pataki-Bittó, F., \& Kapusy, K. (2021). Work environment transformation in the post COVID-19 based on work values of the future workforce. Journal of Corporate Real Estate, Vol. ahead-of-print No. ahead-of-print. Doi: 10.1108/JCRE-08-2020-0031.

Pearson, E. (1929). Some notes on sampling tests with two variables. Biometrika, 21, pp. 337-360. Doi: $10.2307 / 2332565$.

Pearson, E. (1931). The test of significance for the correlation coefficient. Journal of the American Statistical Association, 26. pp. 128-134. Dpi: /10.1080/01621459 .1931 .10503208 .

Peng, M. (2012). Global Strategy. . New York. USA: Free Press.

Rank, O. (2008). Formal structures and informal networks: Structural analysis in organizations. Scandinavian Journal of Management, 24 (2), pp. 145-161. Doi: 10.1016/j.scaman.2008.02.005.

Rigdon, E. (1996). CFI versus RMSEA: A comparison of two fit indexes for structural equation modeling, Structural Equation Modeling. . A Multidisciplinary Journa, 3(4), 369-379. Doi: 10.1080/10705519609540052.

Rothan, H., \& Byrareddy, S. (2020). The epidemiology and pathogenesis of coronavirus dis- ease (COVID-19) outbreak . Journal of Autoimmunity, 109(102433), pp. 1-4. Doi: 10.1016/j.jaut. 2020.102433.

Sallán, J. (2006). Caracterización de los grupos informales de transmición de conocimiento mediante el análisis de redes sociales. Intangible Capital, 11 (2). pp 21-36. .

Satorra, A., \& Bentler, P. (1994). Correction to test and standar errors in covariance structure analysis. En A. V. Eye, \& C. C. (Eds.), Latent Variables Analysis, Applications to Development Research (págs. pp. 399419). CA: University of California, Los Angeles. USA.: Thousand Oaks.

Schabek, T. (2020). The financial performance of sustainable power producers in emerging markets. Renewable Energy, 160, pp. 1408-1419. Doi: 10.1016/j. renene.2020.06.067. 
Schendel, D., \& Hoper, C. (1979). Strategic Management. . Boston. USA: Little, Brow.

Schneider, B. (2000). Some relationships between job satisfaction and organizational. En N. \&. in Ashkanasy, Handbook of Organizational Culture and Climate (págs. pp. 17-21.). Thousand Oaks, CA,: Sage.

Schneider, B., \& Bartlett, C. (1970). Individual difference and organizational climate II: Mensurement of organizational climate by the multi-trait, multi-rater matrix. Personal Psichology, 23. pp. 493-512. Doi: 10.1111/j.1744-6570.1970.tb01368.x.

Senge, P. (1992). Building learning organizations. . Journal of Quality and Participation, 15 (2), 30-38.

Sitar, A., \& Škerlavaj, M. (2018). Learning-structure fit part I. The Learning Organization, pp. 1-12. Doi: 10.1108/ tlo-09-2015-0050 .

Sobel, M.(1987). Direct and indirect effects in linear structural equation models. . Sociological Methods and Research, 16, 155-177. Doi: 10.1177/0049124187016001006.

Soto, G. (4 de 11 de 2020). El Financiero. Obtenido de La otra tragedia: 10 mil empresas y negocios desaparecen por COVID-19: https://www.elfinanciero.com. $m x /$ bloomberg-businessweek/la-pandemia-vino-a-devorarlas-10mil-empresas-y-negocios-ya-no-estan

Sreenivasan, A., \& Suresh, M. (2021). Modeling the enablers of sourcing risks faced by startups in COVID-19 era. Journal of Global Operations and Strategic Sourcing, Vol. ahead-of-print No. ahead-of-print. Doi: 10.1108/ JGOSS-12-2020-0070.

Stefani, S., \& Torriero, A. (2013). Formal and Informal Networks in Organizations. En I. P. A., M. Squillante, \& J. (. Kacprzyk, Advanced Dynamic Modeling of Economic and Social Systems. Studies in Computational Intelligence (págs. 448. pp. 61-77. Doi: 10.1007/978-3642-32903-6_6). Springer, Berlin, Heidelberg.

Terglav, K., Ruzzier, M., \& Kaše, R. (2016). Internal branding process: exploring the role of mediators in top management's leadership?commitment relationship. International Journal of Hospitality Management, 54, pp. 1-11. Doi: 10.1016/j.ijhm.2015.12.007.

Tucker, L., \& Lewis, C. (1973). A reliability coefficient for maximum likelihood factor analysis. Psychometrika, 38(1). pp. 1-10. Doi: 10.1007/BF02291170.
Uzkurt, C., Kumar, R., Kimzan, H., \& Eminoğlu, G. (2013). Role of innovation in the relationship between organizational culture and firm performance: A study of the banking sector in Turkey. European Journal of Innovation Management, 16(1). pp. 92-117. Doi:10.1108/14601061311292878.

Vargas-Hernández, J., Guerra, E., Bojórquez, A., \& Bojórquez, F. (2014). Gestión Estratégica de organizaciones. Buenos Aires, Argentina.: Ediciones Insumos Latinoamericanos .

Värk, A., \& Reino, A. (2020). Practice ecology of knowledge management-connecting the formal, informal and personal. Journal of Documentation, pp. 1-18. Doi: 10.1108/JD-03-2020-0043.

Viloria, J., Daza, D., \& Pérez, K. (2016). Dinámicas e influencias de los grupos informales en las organizaciones. Ánfora, 23(40), pp. 169-194.

White, L., Currie, G., \& Lockett, A. (2016). Pluralized leadership in complex organizations: Exploring the cross network effects between formal and informal leadership relations. The Leadership Quarterly, pp.1-18. Doi: 10.1016/j.leaqua.2016.01.004.

Whiting, K. (21 de 10 de 2020). La mayoría de los líderes piensan que el trabajo a distancia afectará a la productividad, esta es la razón. Recuperado el 5 de 11 de 2020, de https://es.weforum.org/agenda/2020/10/ la-mayoria-de-los-lideres-piensan-que-el-trabajo-a-distanciaafectara-a-la-productividad-esta-es-la-razon/

Wu, X., Nethery, R., Sabath, B., Braun, D., \& Dominici, F. (2020). Exposure to air pollution and COVID-19 mortality in the United States. MedRxiv, pp. 1-36. Doi: 10.1101/ 2020.04.05.20054502.

Xiong, L., \& King, C. (2018). Too much of a good thing? Examining how proactive personality affects employee brand performance under formal and informal organizational support. International Journal of Hospitality Management, 68. pp. 12-22. Doi: 10.1016/j. ijhm.2017.09.007.

Yiing, L., \& Ahmad, K. (2006). The moderating effects of organizational culture on the relationships between leadership behaviour and organizational commitment and between organizational commitment and job satisfaction and performance. Leadersship \& Organization Development Journal, 30 (1). pp. 53-86. Doi:10.1108/01437730910927106.

Younger, S., \& Fisher, G. (2020). The exemplar enigma: New venture image formation in an emergent organizational category. Journal of Business Venturing, 35(1), pp. 1-18. Doi: 10.1016/j.jbusvent.2018.09.002. 